\title{
Engineering of Ultrafast High Efficiency Light-Harvesters
}

\author{
Andreas Albrecht ${ }^{a}$, Julia Nowak and Peter Jomo Walla ${ }^{a}$ \\ Nature provides evidence that there is no fundamental limit for harvesting and funneling nearly all scattered sun-photons \\ onto smaller conversion centers by ultra-fast emergy transfer processes. Recently, a proof-of-principle study showed that \\ this can also be achieved by artificial systems containing light-harvesting pools of randomly oriented molecules that funnel \\ energy to individual, aligned light-redirecting molecules.
}

\begin{abstract}
However, capturing the entire solar spectrum requires engineering of complex multi-element structures considering macroscopic refraction and wave guiding of different spectral ranges of multijunction photovoltaics as well as ultrafast, nanoscopic light-harvesting, energy transfer and funneling, anisotropic absorption and emission and the spectra of a multitude of pigments of different orientations and concentrations. So far, no tool excited that allowed model such structures in one system.
\end{abstract}

Here we present a ray tracing tool allowing to model and analyze such multi-scale structures, including molecular, ultrafast energy transfer and funneling as well as anisotropic absorption and emission as well as micro-and macroscopic waveguiding and raytracing in one tool. We present first results of solar concentrator architectures with the highest theoretical energy conversion efficiency reported so far.

A novel tool is provided that allows to construct, model and analyze any desired complex ultrafast lightharvesting/photovoltaic architecture with the highest efficiencies by considering molecular, nanometric energy transfer and funneling as well as microscopic waveguiding and raytracing.

\section{Introduction}

In the past decade the importance of renewable energy technologies has increased significantly due to the limited amount of fossil fuels and climate concerns. In this context solar energy plays a crucial role since the sun provides approximately 10.000 times as much energy as we consume each year ${ }^{2}$. However, it is challenging to harvest the sun's energy in an efficient way since most photovoltaic cells are limited by the Shockley-Queisser limit. The most efficient multijunction solar cells achieve efficiencies up to $\sim 47 \%{ }^{3}$. However to achieve efficiencies that high, very expensive and rare materials are needed making a large-scale application prohibitively expensive. To circumvent this problem different systems for the concentration of sunlight have been proposed one of which is the luminescent solar concentrator (LSC). This would allow to concentrate also diffusively scattered light from large areas using affordable material onto much less of the precious energy converting material. LSCs are usually polymer structures doped with a fluorescent material. After light is absorbed and reemitted by the fluorescent material a fraction of the light remains trapped in the polymer due to total internal reflection (TIR). The trapped light is subsequently guided towards the edge of the polymer structure where a photovoltaic or another energy converting system can be mounted.

A lot of research has been done to improve the efficiencies of LSCS 4,5 , however in conventional LSCs several intrinsic loss mechanisms greatly reduce the light harvesting efficiency, preventing a widespread practical application. Among the most important intrinsic loss mechanisms are 1. so-called escape-cone-losses, 2. reabsorption losses and 3. thermal losses (Figure 1 a).
1. Escape-cone-losses are caused by photon emission of the lightharvesting pigments in directions where no TIR occurs (green cone in Figure 1 a). In LSCs this problem is of particular importance as primarily fluorophores with transition dipole moments perpendicular to the light's incident direction are excited (photoselection). Since fluorophores primarily emit radiation perpendicular to their transition dipole moment the reemission will preferably occur at directions similar to the incident direction. Due to the fact that most of the sunlight impinges at angles close to perpendicular to the surface, this angle range is also where most of the reemission occurs. The trapping of radiation by TIR, however, only occurs at shallow angles between the light and the surface, thus photoselection leads to a large percentage of photons escaping the waveguide. Typically $30 \%$ of photons are lost in the escape-cone of common LSCs when a polymer host with a refractive index of 1.5 is used ${ }^{6}$.

2. In order to capture all light, obviously also a certain concentration of light-absorption pigments is necessary. However, the necessary high concentrations intrinsically lead to multiple reabsorption events before the light can reach the energy converting device. Each time a fluorophore unnecessarily absorbs and re-emits photons, energy can be lost due to imperfect re-emission yields (fluorescence quantum yield) or directions (escape-cone losses) which quickly sum up to the loss of the major fraction of the originally captured solar energy. In addition, even fluorophores with high fluorescence quantum yields tend to have decreased re-emission yields at high concentrations due to phenomena summarized under the term concentration quenching.

3. The losses that occur when the energy of the originally absorbed photons is higher than that of the re-emitted photons (Stokes-Shift) 
or the band-gap of the used photovoltaic material (ShockleyQueisser limit) can be summarized under the term thermal losses (Figure 1a, right). For example, in silicon solar cells the band gap corresponds to an energy that is less than half of the energy of the major fraction of sunlight photons and therefore thermal losses are responsible for a very large fraction of all losses. This stresses that an optimized light-harvesting and energy conversion efficiency can only be reached when thermal losses are as small as possible in the lightfunneling (small Stokes shift) as well as energy converting device (Band gap matching with incident photon energy).

In our previously published study ${ }^{1}$ we presented a system that is capable of minimizing all these intrinsic loss mechanisms in a simple but very effective concentrator architecture (Figure 1b). It consists of a relatively large amount of randomly oriented light-harvesting donor dyes (green in Figure 1b). The donor's random orientation allows the absorption of light originating from all possible incident angels and its high concentration allows for absorbing all photons of the corresponding spectral range. The donors then funnel the absorbed light on ultrafast timescales and with near to unity quantum efficiency to a smaller population of aligned, lightredirecting acceptors (red in Figure $1 \mathrm{~b}$ ).

The alignment of the light-redirecting acceptors results in emission of the photons in preferential directions perpendicular to their transition dipole moment. This allows efficient redirection of most of the photons into an angle range suitable for highly efficient TIR wave guiding onto a high performance photoconversion device. Escape cone losses below $10 \%$ can easily be achieved $1,6-10$. This ultrafast funneling light re-direction approach is also much more affordable than the use of dichroic coatings ${ }^{11}$ and greatly reduces the number of photons hitting the photoconversion device under unfavorable large angles that usually lead to low conversion efficiencies.

In addition, reabsorption losses are greatly reduced because only small concentrations of the light-redirecting acceptors are necessary. Since acceptors in energy transfer systems generally emit light at lower energies ${ }^{1,12}$ its wavelength does not match the absorption spectrum of the higher concentrated donors. Even a very small energy (Stokes) shift of the low concentrated acceptors allows a significant reduction of re-absorption losses.

To minimize thermal losses the shifts in the emission of lightredirecting acceptor pigments should be as small as possible and the emission itself should be as close as possible to the band gap of the photovoltaic material (Figure 1b, right). Minimization of thermal losses implies also that an optimized system intrinsically requires the use of stack structures that cover several smaller spectral ranges with ideal light-harvesting pigment compositions and photovoltaic band gaps $^{4,10,11,13}$ for each spectral range (figure $1 \mathrm{c}$ ).

The construction of the best ultrafast light-harvesting and energy converting architectures requires the optimization of the spectral properties of the used pigments for each spectral range and match them with the best photovoltaic or energy converting materials currently available. In an optimized stack structure each layer should be capable to direct the fraction of the solar spectrum exactly matching with the band gap of the corresponding high-efficiency material with minimized escape-cone, thermal and re-absorption losses. Minimizing reabsorption losses requires a very careful balance of the structures overall dimensions. Larger collection surfaces improve the geometric yield, i.e. the ratio between harvesting and PV surface, but also increase reabsorption and other losses. Capturing all spectral ranges covered by such multi-junction photovoltaics requires engineering of complex multi-element structures of different size and shape while simultaneously considering macroscopic refraction, reflection and wave guiding as well as molecular light-harvesting, energy funneling, anisotropic absorption and emission. The spectra of a multitude of pigments of different orientations and concentrations have to be taken into account. For the development of such structures it is necessary to have access to tools that consider all parameters governing the overall efficiency and to be able to analyze the principle loss mechanisms of different structures. So far, no such tool was available.

Thus, we here present such a tool to engineer, model and analyze such complex structures including molecular orientation as well as ultrafast high efficiency energy transfer and all other relevant parameters mentioned above. It allows the in-silico optimization of a wide range of such arbitrary structures considering all necessary molecular parameters based on ray tracing Monte-Carlo simulations $10,11,14-17$.

Using these ray tracing simulations the best architecture we found so far is theoretically capable of collecting approximately $60 \%$ of the energy that would be usable by a state of the art quadruple junction solar cell ${ }^{3}$ with a geometric yield of 10 . This represents an architecture with the highest theoretical lightharvesting and energy conversion efficiency reported so far.

\section{Methods}

\section{Description of the used program}

The raytracing-program was custom-built in Python 2.7 using standard numerical libraries ${ }^{18}$. The program is capable of following a photon through any previously defined, customized geometry. The program can handle mirrors, interfaces between two different previously defined materials and detectors where the photons and their energies are registered. In particular it considers also molecular parameters such as energy transfer depending on various local dye concentrations and orientations as well as spectral properties in the absorption and emission of the different pigments. It also considers the band gaps of current record multi junction photovoltaic cells. A demonstration can be seen in the attached Supplementary Movie 1.

When solar irradiance is to be simulated, the photons' wavelengths are drawn from a distribution corresponding to the AM1.5G spectrum ${ }^{19}$ otherwise the wavelength is defined at a fixed value. We use the AM1.5 global tilt spectrum as it is the most commonly 
used solar spectrum in simulations concerning solar concentrators and solar cells. Since we simulate photons individually the spectrum has to be converted to photon flux instead of energy flux. Depending on the performed simulation the direction of propagation is either perpendicular to the simulated LSC's surface or distributed isotropically within the hemisphere pointing towards the surface. The starting point is in all cases simulated to be equally distributed over the surface of the LSC. The polarization vector of the light is, in the beginning, randomly distributed in a plane perpendicular to the propagation direction.

If the photon encounters a mirror it is reflected according to the law of reflection. At an interface the photon can also be reflected, however the interface can also be crossed. The probability for each event is calculated from Fresnel's equations. If the photon crosses the interface it is refracted according to Snell's law and the respective refractive indices.

If a photon crosses into a material in which fluorophores are present a random free-path length left to absorption is calculated. It is drawn from an exponential distribution according to LambertBeer's law:

$$
A=\exp (-\epsilon(\lambda) \cdot \ln (10) \cdot c \cdot l)
$$

In our case the concentration is split into two since we have two different dyes in the system. Also we take the orientation of the dyes into account since the acceptor molecules are aligned within the structure. Thus we have:

$$
A=\exp \left(-3 \cdot \ln (10) \cdot l \cdot\left(\epsilon_{D}(\lambda) \cdot c_{D}+\epsilon_{A}(\lambda) \cdot c_{A}\right.\right.
$$

$$
\left.\left.\cdot \Psi_{A}\right)\right)
$$

Here the indices $D$ and $A$ describe the respective parameters for the donor and the acceptor. The additional factor $\Psi_{A}$ takes the preferential orientation of the acceptor molecules into account. We use an expression similar to Tummeltshammer et al. ${ }^{10}$ to calculate this factor, however if we assume a perfect orientation in our simulations the expression simplifies to:

$$
\Psi=\left|\vec{n}_{\mu}(\theta, \phi) \cdot \vec{n}_{E}\right|^{2}
$$

Where $\vec{n}_{\mu}(\theta, \phi)$ respresents the molecule orientation while $\vec{n}_{E}$ represents the electric field vector of the light.

If the photon has to travel further than the calculated free path length within the absorbing medium it is said to be absorbed after having traveled the free path distance. Within the absorbed state several processes compete with each other: 1 . The photon can be reemitted at a wavelength drawn from the fluorescence spectrum of the excited dye. 2. It can be lost due to internal conversion. 3 . Energy transfer can occur if a second dye is present and the spectral overlap between the respective absorption and emission spectra is sufficiently big. Depending on the probabilities of each process one pathway is chosen randomly.
The probability for energy transfer has been calculated as follows. First, the FRET overlap integral between the absorption spectrum of the acceptor and the emission spectrum of the donor has to be calculated:

$$
R_{0}^{6}=\frac{9 \ln 10 \cdot \kappa^{2} \Phi_{0}}{128 \pi^{5} n^{4} N_{A}} \int F_{D}(\lambda) \epsilon_{A}(\lambda) \lambda^{4} d \lambda
$$

Where $\Phi_{0}$ is the quantum yield of the donor without energy transfer, $\mathrm{n}$ is the refractive index, $F_{D}(\lambda)$ is the normalized fluorescence spectrum of the donor and $\epsilon_{A}(\lambda)$ is the wavelength dependent extinction of the acceptor. We approximate the angles between donor and acceptor to be statistically distributed so we assume $\kappa^{2}=\frac{2}{3}$.

From this integration the FRET radius can be determined. From the FRET radius $R_{0}$ and the acceptor concentration the energy transfer probability can be approximated as follows ${ }^{20}$ :

$$
\Phi_{E T}=1-e^{-\alpha \frac{c}{c_{0}}}
$$

Where

$$
c_{0}=\frac{3000}{4 \pi N R_{0}^{3}}
$$

and $\alpha=1.42$.

We did not notice significant differences between the results obtained with this equation compared to a calculating the individual energy transfer efficiencies in a spherical volume with the radius $10 R_{0}$ around the donor. Since the simulation of many individual molecules would take much more time we used the above approximation for our calculations.

The emission then occurs either isotropically or according to a cosine-squared distribution perpendicular to a previously specified orientation axis depending on whether the emitting dye is aligned or not.

The basis for the used spectra $F_{D}(\lambda), \epsilon_{A}(\lambda)$ and $F_{A}(\lambda), \epsilon_{D}(\lambda)$ was taken from the literature ${ }^{21-23}$. For simplicity we used the spectra of Coumarin 1 and Coumarin 6, the dyes used in our previous experimental study ${ }^{1}$, as a basis for the used spectra. The long wavelength tail of the absorption spectra is particularly important for our simulations since most reabsorption events will take place at these wavelengths. However, determining this very low absorption with very high accuracy is experimentally difficult, even with the best state of the art absorption spectrometers. As to not underestimate the reabsorption effects we extrapolated the spectra by fitting exponential decay functions to the noisy tail in these absorption spectra. Values below $2 \mathrm{e}-4$ times the peak value were set to this value until being set to zero at 1.5 times the wavelength of the peak maximum. This approximation is also made as to not underestimate reabsorption effects. The approximated value of $2 \mathrm{e}-4$ is based on measurements performed by Wilson et al. 
${ }^{24}$ who measured the long-wavelength tail absorption of dyes in solar concentrators with high accuracy.

We simulate a structure containing several dyes with spectra spanning large parts of the solar spectrum. All used spectra, are hypothetical and were derived from the real dyes' spectra. We used the basis of the Coumarin's spectra to yield optimized spectra with wider absorption bands in the donor dye and narrower emission bands in the absorption dye. These manipulations of the used spectra are similar to regular dyes' or quantum dot spectra so they are suitable for the study of the proposed structure's theoretical limits. Also, as we are interested in the hypothetical upper limits of physically possible architectures, we chose to use fluorescence quantum yields of $100 \%$ and $99 \%$ for the dyes. Similarly high values have been reported, for example, for dyes ${ }^{25}$, in particular when they are embedded in polymers ${ }^{26}$, or also for quantum dots ${ }^{11}$.

The Monte-Carlo simulations were performed using 100.000 realizations if not denoted otherwise. Please note that some of our systems consist of element numbers orders of magnitude higher than previously reported structures and therefore the number of calculated photons are sometimes lower compared. However, 100 repetitions of quantum efficiency calculations for different concentrations demonstrate that the efficiencies reported here have a standard deviation of about $0.5 \%$ when 100.000 photons were simulated. In the future, multiple core approaches can be developed to compute even higher numbers in a shorter amount of time.

We present three different parameters to quantify the efficiency of the simulated systems. The first quantity is the light harvesting quantum efficiency, $\Phi_{Q E}$, which is the total number of photons hitting the different photovoltaics divided by the total number of photons incident on the light-harvesting architecture. The second is the energy harvesting efficiency, $\Phi_{E E}$, which is the ratio of the total energy hitting the photovoltaics divided by the overall incident energy.

However, even state of the art multi junction photovoltaic cells cannot make use of any photons with an energy below the lowest band gap (See Figure $1 \mathrm{c}$ ) and photons hitting any PV material with energies below the band gap of the corresponding layer must not be considered in the overall light-harvesting efficiency. Therefore, one of the most important parameters for high-efficiency lightharvesters is the fraction of energy from photons that hit the correct PV material, $i$, with the correct band gap energy fitting to the photons energy. To account for this the actual $P V$ usable energy efficiency, $\Phi_{P V U}$, is calculated. For the fundamental reasons described above, the maximum energy that can be generated from a photon hitting the correct PV layer is determined by the band gap energy of that layer, $E_{\text {gap }}^{i}$. According to this, the maximum energy that a multi-layer light-harvesting architecture cell can generate is the sum of band gap energies for all photons, $n_{L H}^{i}$, hitting their corresponding, correct photovoltaic layer $i$ :

$$
\begin{gathered}
E_{L H}^{\text {sum }}=n_{L H}^{1} \cdot E_{\text {gap }}^{1} \\
+n_{L H}^{2} \cdot E_{\text {gap }}^{2}+n_{L H}^{3} \cdot E_{\text {gap }}^{3} \\
+n_{L H}^{4} \cdot E_{\text {gap }}^{4}
\end{gathered}
$$

In a light harvesting architecture without any losses this energy would be identical to the maximum energy that a multijunction photovoltaic cell without any light harvesting architecture can generate. The corresponding maximum energy of a multijunction photovoltaic cell is the sum of band gap energies for all photons of the incident light that have a sufficient photon energy, $n^{i}$, for the corresponding photovoltaic layers $i$ :

$$
\begin{gathered}
E^{\text {sum }}=n^{1} \cdot E_{\text {gap }}^{1} \\
+n^{2} \cdot E_{\text {gap }}^{2}+n^{3} \cdot E_{\text {gap }}^{3} \\
+n^{4} \cdot E_{\text {gap }}^{4}
\end{gathered}
$$

The PV usable energy efficiency is the fraction of the photon energies that is still correctly directed via a light-harvesting architecture to the different PV layers compared to a direct illumination of the PV:

$$
\Phi_{P V U}=\frac{E_{L H}^{\text {sum }}}{E^{\text {sum }}}
$$

In the present study, the values reported in Tibbits et.al. ${ }^{3}$ were used for the band gap energies $E_{\text {gap }}^{i}$. Increasing band gap energies shall correspond to increasing $i$, i.e. the lowest band gap energy is $E_{g a p}^{1}$. Of course any other band gap energies of future photovoltaic materials could also be used. The photon numbers $n^{i}$ were determined from counting all photons in the incident light with a photon energy falling in the range between $E_{g a p}^{i}$ and $E_{\text {gap }}^{i+1}$ or $>E_{\text {gap }}^{4}$ in the case of $n^{4}$. The photon numbers, $n_{L H}^{i}$, were determined from counting all photons hitting a distinct photovoltaic layer, $i$, with a photon energy $>E_{\text {gap }}^{i}$ of that layer.

To validate our raytracer we simulated the simplest systems previously published by Tummeltshammer et al. ${ }^{10}$. The results are shown in supplementary figure 1 . These results are in very good agreement with the previously published results. In addition, we simulated our previously reported experimental system [6] and optimized the donor and acceptor concentrations for exactly this system in silico (cf. 
Supplementary Figure 2). Also for this system, nearly identical values as empirically optimized in the experiments were observed.

\section{Single layer structure}

For our previous study we created two-dye LSC foils where the first dye is isotropically oriented to collect light coming from all directions. The energy from the first dye is then transferred to the aligned second dye where it can be emitted in a preferential direction.

First we use a simple rectangular structure (figure 2 a) to optimize the concentrations of the used pigments. The results from these calculations are then used in more complex structures in which a systematic optimization of the concentrations is computationally more expensive. In this structure we explore the light-funneling capabilities by simulating monochromatic illumination at the donor's peak absorption wavelength perpendicular to the LSC's surface. We optimize the donor and acceptor concentrations for different dye systems that absorb and funnel light at different wavelengths.

We apply these results to a more complex geometry similar to the one previously proposed. In this system light is funneled towards a conversion center by arranging multiple foils in a larger system in a lamella-type structure ${ }^{1}$. In the simulation presented here, the foils are cut into small cuboids that are stacked to a stair-like structure and embedded into a polymer as depicted in figure $2 \mathrm{~b}$.

Since rather high acceptor concentrations are needed to achieve efficient energy transfer we try to limit excessive reabsorption by using this stair structure. In this structure the average concentration is held low enough to avoid reabsorption while the local concentrations are high enough to achieve efficient FRET. The cuboids are embedded into a polymer with an identical refractive index so effectively no refraction occurs at the interfaces and light can be trapped in the individual layers. Also, this way the light is not trapped in the stair structure which would, again, lead to higher reabsorption. We simulate the bottom of the structure to be a mirror while one wall of the structure is an index-matched PV-Cell. An accurate representation of the actual simulated structure, including exemplary calculated photon trajectories, is shown in figure $2 \mathrm{~b}$. Obviously, a bigger PV-Cell is needed for our stair structure when compared to simpler rectangular structure with the same amount of collection surface and dye-doped material. However, to achieve a similar efficiency the cells would have to be very small, requiring a large number of small PV-Cells which would make the practical use of this concept difficult. Thus, our proposed structure offers a solution to building larger systems while the dye concentrations are reasonably high by shifting the collection material into different planes so only one bigger PV-Cell is needed. Also, in our previous study, the orientation of the dyes was achieved by stretching the dye-doped polymers. Using this technique, the dyes are oriented in a way that requires the manufactured foils' long axis to be parallel to the collection geometry which can easily be realized by using the presented stair structure.
In the first proposed stair-geometry the LSC has a $2 \mathrm{~cm} \times 2 \mathrm{~cm}$ collection surface and a height of $2 \mathrm{~mm}$. The cuboids that are arranged in the stair shape are $181 \mu \mathrm{m}$ in height and $50 \mu \mathrm{m}$ in width (cf. Figure 2 b).

\section{Multiple stacked layer structure}

Our next goal is to collect as much sunlight as possible. As previously mentioned, this can be only achieved by using stack structures that cover several smaller spectral ranges with ideal light-harvesting pigment compositions and photovoltaic band gaps. In the present study we decided to use multiple layers of the previously described stair structure separated by an air gap, each with a different pair of dyes (cf. figure 1c). The use of several layers allows us to collect large portions of the solar spectrum and also splits the solar spectrum spectrally. Also, only a small amount of each photon's energy is lost in each layer as the Stokes-Shifts do not have to be as high as in a one-layer structure where only one dye is used to collect the entire solar spectrum (Compare Figure 1 a with Figure c).

When the solar spectrum is spectrally separated by the individual layers in the multi-layer concentrator structure, it is not necessary to use a full quadruple junction solar cell as PVCell for each layer. Rather, only the individual components of the cell in the layer responsible for the collection of the corresponding part of the spectrum needs to be used. I.e., even if in a quadruple light-harvesting structure the PV area is four times larger (see figure $1 \mathrm{c}$ ), this actually corresponds only to a quarter of this area of a full quadruple PV-cell. This must be considered when the geometric factors of different architectures are given.

As described previously we use the Coumarin 1/Coumarin 6 system as a base system from which we derive the other dye systems by altering their absorption and fluorescence spectra. We shifted, widened and narrowed the emission and absorption bands to match the desired spectral properties keeping all of these operations within realistic physical and experimental limits. Wide absorption bands combined with narrow emission bands, as chosen for the donors, are common in quantum dots, while rather narrow absorption and emission spectra can often be found in organic dyes. We tried to tune the acceptor's fluorescence spectra to the band gaps of the PV-Cell reported by Tibbits et al. 3 to have the possibility of combining our system with the previously reported cells.

As previously described, the donor and acceptor concentrations were first optimized in the simplified structure (figure 2 a) individually for each layer to find approximate values for the optimum concentrations and by using monochromatic excitation. It is not useful to optimize these parameters in the individual layers using the sun spectrum since excessively high optimum concentrations would be found due to higher concentrations leading to a broader bandwidth of effective absorption of the solar spectrum. In the full stack structure (figure $3 \mathrm{a}$ and $\mathrm{b}$ ) almost the entire spectrum is covered by high values in the absorption bands of the dyes altogether. High dye concentrations in the individual layers are not needed to cover a broad bandwidth, however they would lead to excessive reabsorption thereby limiting the collection efficiencies.

The optimization for the individual layers is carried out by calculating single points combining different donor and acceptor concentrations. 
For a first optimization in the full stack structure we used the simplified geometry (figure 3 a) and used random concentrations of each dye for the entire system.

\section{Results}

Supplementary Movie 1 demonstrates how the software generally works and in Figure 4 a an optimized structure is illustrated along with the best optimized spectra we found so far (figure $4 \mathrm{~b}$ ). Also shown are the External Quantum Efficiency (EQE) spectra of a state of the art quadruple junction solar cell (black, from left to right GalnP, GaAs, GalnAsP, GalnAs, taken from Tibbits et al. ${ }^{3}$ ). In addition, Figure 5 shows the wavelength histograms of all photons hitting the different photovoltaic layers.

To approach optimized concentrations, we first simulated individual layers in the simplified geometry (cf. figure 2 a) using monochromatic illumination at the donor's absorption maximum perpendicular to the collector's surface and varied the concentrations of the donor and the acceptor systematically (Supplementary Figure 3). For these special conditions we observed well defined optima in all layers that could then serve as starting point for the concentration optimization in the more complex structures and considering the entire spectral range of the sun-light. For all layers, overall light-harvesting quantum efficiencies, $\Phi_{Q E}$, that include absorption, energy transfer, emission, lightredirectioning and waveguiding efficiencies, of around $80 \%$ were observed. They were also rather insensitive to small changes in the concentrations as long as the concentrations are high enough to absorb most of the incoming light and allow for efficient FRET. When fluorescence quantum efficiencies of $\Phi_{F l}=99 \%$ are used instead of $100 \%$ for both pigments the light-harvesting quantum efficiencies, $\Phi_{Q E}$, are approximately $2 \%$ lower in all cases. The finding that the overall light-harvesting quantum efficiency decreases approximately two times more indicates that approximately one reabsorption event per photon occurs in the presented geometry.

Next we optimized the four-layered structure using the entire spectral range of the sunlight instead of using only monochromatic illumination for each single layer. Again, a simplified structure consisting of simple rectangular shapes (figure 3 a) was first investigated. For the optimization of the concentrations we simulated efficiencies for random sets of concentrations in a similar range as previously observed in the more simple single layer structures. The random concentrations were chosen between 0 and $20 \mathrm{mM}$ for the donor and between 0 and $10 \mathrm{mM}$ for the acceptor. Since we use here the entire solar spectrum instead of only monochromatic radiation at the peak of the donor absorption, the concentration optimal for harvesting also light at wavelengths outside this peak is expected to be larger. Therefore, the interval for the donor concentrations was chosen to be somewhat larger.
We investigated the efficiencies by using two types of incident radiation angle distributions: straight illumination perpendicular to the collection surface and isotropic illumination coming from equally distributed random incident directions (Figure 6). Real illumination conditions will correspond to situations in between these extreme cases - in most cases even diffusive illumination still has some remaining preferential direction of the incoming light. The concentrations in the system were first optimized using perpendicular illumination. The same concentrations were then used to test the system also under isotropic illumination conditions.

For the different conditions the resulting efficiencies are shown in figure 6 . As mentioned above, we used three different quantities to assess the overall efficiency of the system optimized in spectra, concentrations and macroscopic dimensions for all layers. The light harvesting quantum efficiency $\Phi_{Q E}$ represents the number of photons hitting the photovoltaics divided by the total number incident photons, the overall energy efficiency $\Phi_{E E}$ is the total energy hitting the photovoltaics divided by the overall incident energy and the $P V$ usable energy efficiency $\Phi_{P V U}$ is the total fraction of energy hitting the photovoltaic from photons of different wavelengths that can actually be converted considering the band gaps of the different PV layers (see methods section for more details).

The white bars corresponds to end-to-end efficiencies, including all losses as well as reflection losses on the first surface that is usually removed by anti-reflection coatings. The black bars corresponds to efficiencies excluding these first surface reflection losses.

As expected, out of the three observed quantities the light harvesting quantum efficiency $\Phi_{Q E}$ is the biggest as, intrinsically, quantum efficiencies do not contain thermal losses. However, in most cases it is rather close to the overall energy efficiency $\Phi_{E E}$. This result underlines that the thermal losses in the system are already quite optimized.

As long as reflection losses on the first surface are not minimized efficiencies for straight illumination are, in all cases, higher than those for isotropic illumination (white bars). This is owed to the fact that in the case of isotropic illumination approximately $23 \%$ of incoming photons are reflected at the surface while only $4 \%$ of incoming photons are reflected when perpendicular illumination is used. However, if these surface losses are excluded (black bars) this difference is greatly reduced.

When the PV-bandgaps are not taken into account the energy efficiencies, $\Phi_{E E}$, are bigger in the isotropic case than the efficiency in the case of perpendicular illumination. When the bandgaps are taken into account, however, the energy efficiencies, $\Phi_{P V U}$, are almost identical. A closer inspection indicates that random photons that have never been absorbed hit the photovoltaics as well. As these photons do not necessarily fit the respective band gap, approximately $9 \%$ of photons hit photovoltaic layers with energies not sufficient for the corresponding band gaps when using isotropic 
illumination whereas only approximately $6 \%$ of photons have too little energy when perpendicular illumination is used.

After excluding the surface losses the remaining loss mechanisms are mainly the reflection between the individual layers, escapecone-losses and thermal losses within the dye and at the bandgaps.

As stated above, in the case of perpendicular illumination, approximately $4 \%$ of all photons are reflected before entering the collection structure. Also, photons with energies too low to be absorbed by the pigments will not be redirected and leave the collector on the same trajectory as they entered. Together, with this effect further reflections on the interfaces between the layers and remaining escape cone losses account for another $30 \%$ of photons being lost. The observation of such loss mechanisms including reflections at interfaces between the layers provide very valuable insights that will significantly help to further minimize such remaining losses in the future.

For example, the surface losses are higher when isotropic illumination is used which increases the total percentage of lost photons to approximately $44 \%$. However, in this case it is easier for photons to be absorbed as they can now also be absorbed by the acceptor. Also, in this case photons can arrive at a PV-Cell without ever being absorbed by a dye. Thus, the percentage of lost photons after excluding surface losses is smaller at approximately $21 \%$.

When the optimized concentrations are used in the stack-stairstructure (figure $3 \mathrm{~b}$ and figure $4 \mathrm{a}$ ) approximately the same efficiencies can be obtained. As our proposed structures require rather high dye concentrations to achieve a high FRET-efficiency all simulated structures are rather small. For example, the square area of the light harvesting structure is $4 \mathrm{~cm}^{2}$ and the individual photovoltaic areas are $0.4 \mathrm{~cm}^{2}$. The stair structure enables an efficient upscaling of this type of concentrator structure requiring only a smaller number of individual PV modules. Here it is important to note that four individual photovoltaic areas of $0.4 \mathrm{~cm}^{2}$ of each spectral range corresponds to the same amount of material as in a quadruple solar cell of only one photovoltaic area of $0.4 \mathrm{~cm}^{2}$. I.e., even though the overall PV area is larger in this light harvesting architecture it still corresponds to only a four times smaller area of a quadruple solar cell. It can be expected that it is even advantageous to split the four materials into separated areas, as this reduces photon absorption effects in previous layers in a stacked structure.

In summary, approximately $60 \%$ of the energy in the full solar spectrum can be redirected by the proposed concentrator structure with isotropic as well as perpendicular illumination and a geometric factor of 10. Even though higher efficiencies might be possible in the future, this represents the highest theoretical concentrator efficacy and would require more than five time less material of current high-efficiency photovoltaic material.
In the present work we introduce a ray tracing tool that allows the engineering of very complex multi scale macroscopic and ultrafast molecular light-harvesting and light-concentrating structures. Structures containing multiple different materials and structures on different molecular as well as macroscopic scales can be entered and computed. The tool considers waveguiding (total internal reflection), refraction and reflection as well as orientation dependent molecular light absorption and emission, funneling energy transfer between different light harvesting molecules with either isotropic or well defined molecular orientations. Additionally, intermolecular distances, different spectral properties of multiple donors and acceptors and multiple layers of light-harvesters and photovoltaics are considered. The resulting light-redirecting efficiencies are then computed and optimized. The tool allows the optimization of these systems with respect to current state-of the art and world-record energy conversion devices such as stacked (multi junction) solar cells. In addition, it offers valuable in silico insight into the most important mechanisms that dominate the remaining losses.

Using this tool, the best architecture we found so far can redirect approximately $60 \%$ of the solar energy usable by current world record quadruple junction PV cell at an effective concentration factor of 10 . To the best of our knowledge this is the highest theoretical energy concentrating value for current quadruple junction energy converters reported so far.

As we optimized the spectral properties of hypothetical, but physically feasible, molecules for our study, it is now necessary to find actual real pigments to construct devices engineered by our in silico approach.

Further optimization of these structures is in progress and even higher efficiencies are likely. As our tool can calculate very complex structures, it is ideally suited to engineer such structures in the future.

\section{Conflicts of interest}

The University of Braunschweig and P.J.W. filed a patent for parts of this work. The remaining authors declare no competing financial interests.

\section{Acknowledgements}

This work was supported by generous grants from the German science foundation (Deutsche Forschungsgemeinschaft (DFG) (INST 188/334-1 FUGG, GRK2223))

\section{Notes and references}

1 A. Pieper, M. Hohgardt, M. Willich, D. A. Gacek, N. Hafi, D. Pfennig, A. Albrecht and P. J. Walla, Nature Communications, , DOI:10.1038/s41467-018-03103-4.

2 G. W. Crabtree and N. S. Lewis, Physics Today, 2007, 60, 37-42.

\section{Conclusions}


3 T. N. D. Tibbits, P. Beutel, M. Grave, C. Karcher, E. Oliva, G. Siefer, A. Wekkeli, M. Schachtner, F. Dimroth, A. W. Bett, R. Krause, M. Piccin, N. Blanc, M. Muñoz-Rico, C. Arena, E. Guiot, C. CharlesAlfred, C. Drazek, F. Janin, L. Farrugia, B. Hoarau, J. Wasselin, A. Tauzin and T. Signamarcheix, 2014, 4.

4 M. G. Debije and P. P. C. Verbunt, Advanced Energy Materials, 2012, 2, 12-35.

5 R. Mazzaro and A. Vomiero, Advanced Energy Materials, 2018, 1801903.

6 C. L. Mulder, P. D. Reusswig, A. M. Velázquez, H. Kim, C. Rotschild and M. A. Baldo, Optics Express, 2010, 18, A79-A90.

7 R. W. MacQueen, Y. Y. Cheng, R. G. Clady and T. W. Schmidt, Optics Express, 2010, 18, A161-A166.

8 C. L. Mulder, P. D. Reusswig, A. P. Beyler, H. Kim, C. Rotschild and M. A. Baldo, Optics express, 2010, 18, A91-A99.

9 S. McDowall, B. L. Johnson and D. L. Patrick, Journal of Applied Physics, 2010, 108, 053508.

10 C. Tummeltshammer, A. Taylor, A. J. Kenyon and I. Papakonstantinou, Journal of Applied Physics, 2014, 116, 173103.

11 D. R. Needell, O. Ilic, C. R. Bukowsky, Z. Nett, L. Xu, J. He, H. Bauser, B. G. Lee, J. F. Geisz, R. G. Nuzzo, A. P. Alivisatos and H. A. Atwater, arXiv:1710.00034 [physics].

12 C. Tummeltshammer, M. Portnoi, S. A. Mitchell, A.-T. Lee, A. J. Kenyon, A. B. Tabor and I. Papakonstantinou, Nano Energy, 2017, 32, 263-270.

13 B. Fisher and J. Biddle, Solar Energy Materials and Solar Cells, 2011, 95, 1741-1755.

14 S. W. Leow, C. Corrado, M. Osborn and S. A. Carter, ed. A. P. Plesniak, 2013, p. 882103.

15 D. Şahin, B. Ilan and D. F. Kelley, Journal of Applied Physics, 2011, 110, 033108.

16 S. R. Wilton, M. R. Fetterman, J. J. Low, G. You, Z. Jiang and J. Xu, Optics Express, 2014, 22, A35.

17 S. Woei Leow, C. Corrado, M. Osborn, M. Isaacson, G. Alers and

S. A. Carter, Journal of Applied Physics, 2013, 113, 214510.

18 E. Jones, T. Oliphant, P. Peterson and others, SciPy: Open source scientific tools for Python, 2001.

19 AM1.5G Spectrum, https://rredc.nrel.gov/solar/spectra/am1.5/.

20 T. Förster, Discuss. Faraday Soc., 1959, 27, 7-17.
21 H. Du, R.-C. A. Fuh, J. Li, L. A. Corkan and J. S. Lindsey, Photochemistry and Photobiology, 1998, 68, 141-142.

22 J. M. Dixon, M. Taniguchi and J. S. Lindsey, Photochemistry and Photobiology, 2007, 81, 212-213.

23 G. A. Reynolds and K. H. Drexhage, Optics Communications, $1975,13,222-225$.

24 L. R. Wilson, B. C. Rowan, N. Robertson, O. Moudam, A. C. Jones and B. S. Richards, Applied Optics, 2010, 49, 1651.

25 D. Magde, R. Wong and P. G. Seybold, Photochemistry and Photobiology, 2007, 75, 327-334.

26 B. C. Rowan, L. R. Wilson and B. S. Richards, IEEE Journal of Selected Topics in Quantum Electronics, 2008, 14, 1312-1322. 